\title{
Effects of the design parameters of multimodel adaptive control on the performance of a flexible transmission system
}

\author{
Alireza Karimi ${ }^{1, *, \dagger}$, Ioan Doré Landau ${ }^{2}$ and Nader Motee ${ }^{1}$ \\ ${ }^{1}$ Electrical Engineering Department, Sharif University of Technology, Azadi Ave., 11365-9363 Tehran, Iran \\ ${ }^{2}$ Laboratoire d'Automatique de Grenoble (CNRS-INPG-UJF) ENSIEG, BP. 46, 38402 St. Martin d'Hères, France
}

\begin{abstract}
SUMMARY
Design parameters selection in the multimodel adaptive control based on switching and tuning will be investigated. Some design parameters like number of fixed and adaptive models, forgetting factor and minimum time delay between switchings will be considered. A recently developed parameter adaptation algorithm based on closed-loop output error will be compared with the classical least-squares prediction error algorithm in the multimodel adaptive control. The effects of these parameters on the performance in tracking and in regulation of a flexible transmission system will be studied via several simulation examples. Copyright (C) 2001 John Wiley \& Sons, Ltd.
\end{abstract}

KEY WORDS: adaptive control; multimodel; switching; closed-loop identification

\section{INTRODUCTION}

The plants subjected to abrupt and large parameter variations are generally very difficult to control. A classical adaptive controller or a fixed robust controller encounter the difficulties to solve this problem. An adaptive controller is not fast enough to follow the parameter variations and unacceptable transients occur. Whereas a fixed robust controller normally leads to poor performances because of large uncertainties.

A solution based on switching between different controllers for this type of plants has been probably proposed for the first time in Reference [1]. The main problem of switching is to decide when a controller should be switched to the plant. Some authors proposed a predetermined switching sequence [2-4] but the multimodel approach seems more interesting. This approach based on multiple models and switching will allow the transient responses to be improved in the presence of large and fast parametric variations [5-7]. In this approach, we suppose that a set of models for different operating points is a priori known. Then at every instant a controller corresponding to the model yielding the minimum of a performance index is used to compute the

\footnotetext{
* Correspondence to: Alireza Karimi, Institut d'Automatique, DGM, Ecole Polytechnique Fédérale de Lausanne, 1015 Lausanne, Switzerland.

†E-mail: alireza.karimi@epfl.ch
}

Copyright (C) 2001 John Wiley \& Sons, Ltd. 
control input. The precision of the control can be further improved using an adaptive model (a model whose parameters are updated with a parameter adaptation algorithm) in the set of models. This method together with a stability analysis was proposed by Narendra and Balakrishnan [8].

Although it has already been shown [7-9] that the performance of a system can be significantly improved using the multiple model adaptive control based on switching and tuning, the design parameters selection for this approach has not been investigated in details. In this paper, we try to study the effects of some design parameters of this approach on the performance of a flexible transmission system. This system is very interesting because the frequency characteristics of its model change drastically with load and makes it a suitable laboratory set-up for robust and adaptive control. This system has been also the subject of an international benchmark on robust digital control [10].

The design parameters we consider for our study, are

- number of fixed and adaptive models,

- type of parameter adaptation algorithm for adaptive models,

- forgetting factor in the switching rule,

- minimum delay time between two switchings.

The rest of the paper is organized as follows. The flexible transmission is described in Section 2 . The principles of the multiple models adaptive control based on switching and tuning will be presented briefly in Section 3. Section 4 explains the basis of the closed-loop output error (CLOE) parameter estimation algorithm. The simulation results are given in Section 5 and finally, Section 6 presents the concluding remarks.

\section{SYSTEM DESCRIPTION}

We consider the flexible transmission system built at Laboratoire d'Automatique de Grenoble (INPG-CNRS), France, which has been used for a benchmark in robust digital control at European Control Conference in Rome, 1995 [10]. The system consists of three horizontal pulleys connected by two elastic belts (see Figure 1). The first pulley is driven by a DC motor whose position is controlled by local feedback. The objective is to control the position of the third pulley which may be loaded with small disks (maximum 12 disks of $300 \mathrm{~g}$ ). The system input is the reference for the axis position of the first pulley. A PC is used to control the system. The sampling frequency is $20 \mathrm{~Hz}$.

The system has a pure time delay equal to two sampling periods and an unstable zero. The discrete-time plant is described by the following transfer operator:

$$
H\left(q^{-1}\right)=\frac{q^{-\mathrm{d}} B\left(q^{-1}\right)}{A\left(q^{-1}\right)}
$$

where $q^{-1}$ is the backward shift operator, $\mathrm{d}$ is the plant pure time delay and:

$$
\begin{gathered}
B\left(q^{-1}\right)=b_{1} q^{-1}+\cdots+b_{n_{B}} q^{-n_{B}} \\
A\left(q^{-1}\right)=1+a_{1} q^{-1}+\cdots+a_{n_{A}} q^{-n_{A}}
\end{gathered}
$$




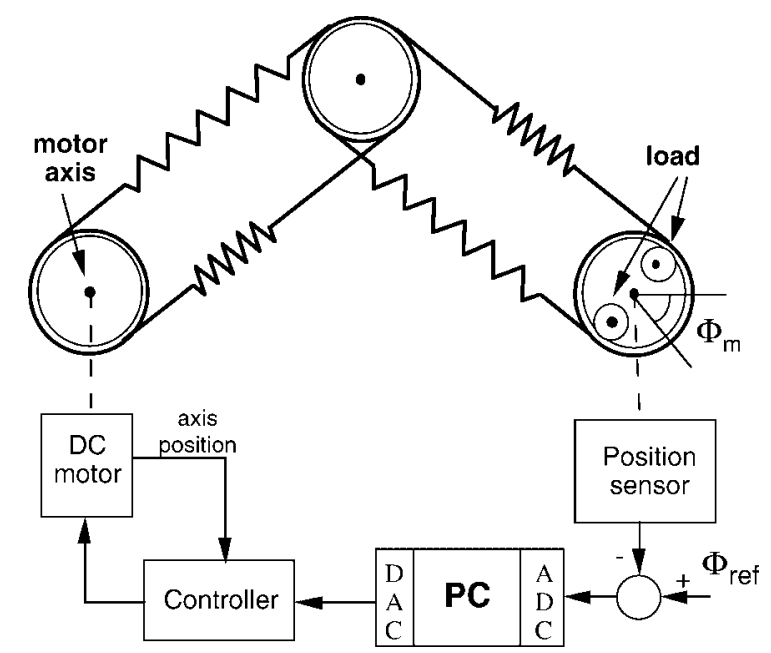

Figure 1. Schematic diagram of the flexible transmission.

Table I. Identified parameters of the plant models in different loadings.

\begin{tabular}{ccccccc}
\hline Model no. & $b_{1}$ & $b_{2}$ & $a_{1}$ & $a_{2}$ & $a_{3}$ & $a_{4}$ \\
\hline 0 & 0.4693 & 0.3957 & -1.3750 & 1.5874 & -1.3168 & 0.9321 \\
1 & 0.3660 & 0.3030 & -1.5767 & 1.7928 & -1.5097 & 0.9337 \\
2 & 0.3040 & 0.2405 & -1.7020 & 1.9155 & -1.6260 & 0.9303 \\
3 & 0.2581 & 0.2311 & -1.7839 & 1.9966 & -1.7025 & 0.9271 \\
4 & 0.2216 & 0.1985 & -1.8498 & 2.0623 & -1.7647 & 0.9302 \\
5 & 0.1956 & 0.1647 & -1.9017 & 2.1211 & -1.8211 & 0.9353 \\
6 & 0.1785 & 0.1529 & -1.9371 & 2.1540 & -1.8490 & 0.9301 \\
7 & 0.1596 & 0.1306 & -1.9700 & 2.1881 & -1.8857 & 0.9348 \\
8 & 0.1448 & 0.1233 & -1.9930 & 2.2099 & -1.9053 & 0.9325 \\
9 & 0.1377 & 0.1183 & -2.0146 & 2.2327 & -1.9247 & 0.9299 \\
10 & 0.1205 & 0.1022 & -2.0390 & 2.2597 & -1.9520 & 0.9374 \\
11 & 0.1153 & 0.1054 & -2.0493 & 2.2656 & -1.9560 & 0.9318 \\
12 & 0.1033 & 0.0850 & -2.0688 & 2.2891 & -1.9816 & 0.9413 \\
\hline
\end{tabular}

The parameters of the 13 identified models numbered from 0 to 12 corresponding to the number of disks on the third pulley are given in Table I. The system is characterized by two low damped vibration modes (with damping factors of less than 0.05 ), subject to a large variation in the presence of load. A variation of about 100 per cent of the frequency of the first vibration mode occurs when passing from the full loaded case (12 disks) to the unloaded case. The magnitude of the frequency responses for three different models (nos. 0,6 and 12) are depicted in Figure 2. The frequencies are normalized with the sampling frequency $(20 \mathrm{~Hz})$.

The flexible transmission system is chosen in order to study the performance of the multimodel adaptive control. Having one unstable zero, pure time delay, very low damped vibration modes and very large parameter variation with load make this system very difficult to control. High 


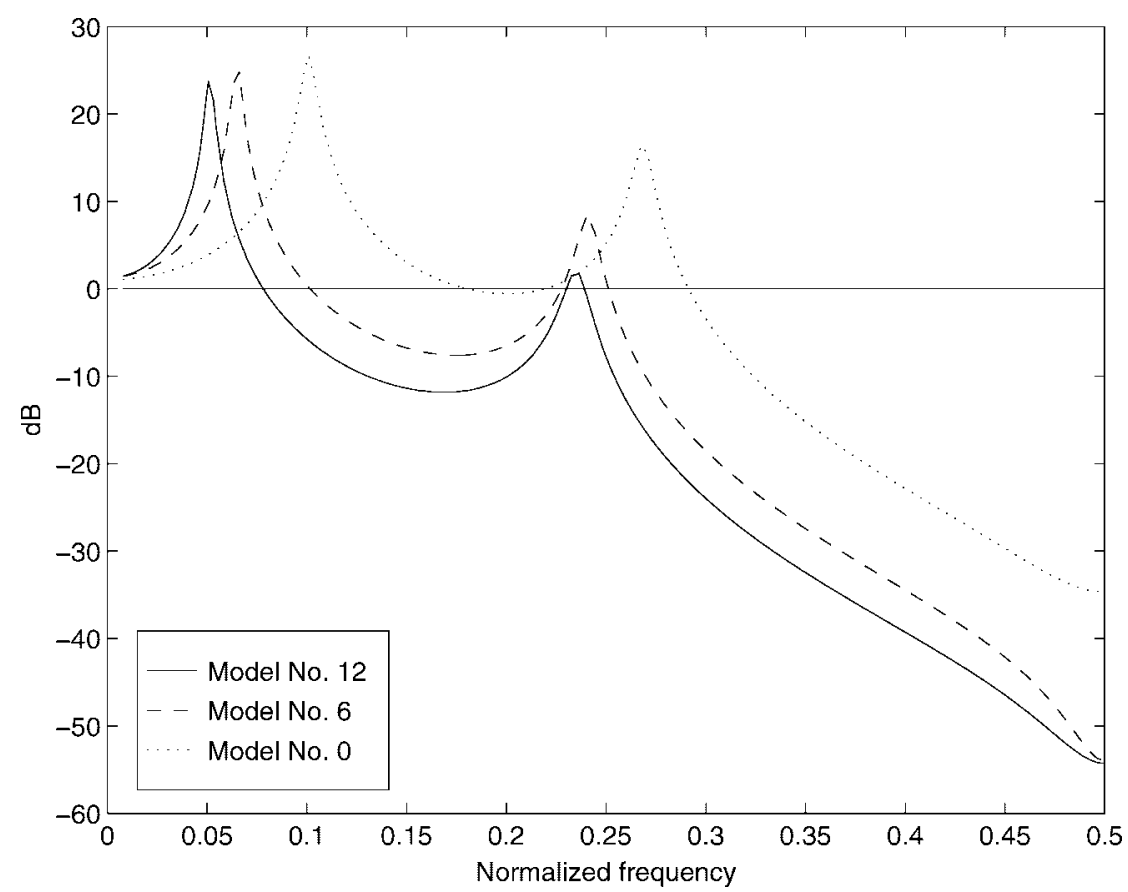

Figure 2. The magnitude of the frequency responses for three different loadings.

performances cannot be achieved using one fixed robust controller and the classical adaptive controllers cannot handle the fast and frequent parameter variation of the system. Therefore, this system may be considered as an interesting benchmark for multimodel adaptive control.

\section{PRINCIPLES OF ADAPTIVE CONTROL WITH SWITCHING AND TUNING}

The main idea of this method is to choose the best model for the plant from an a priori known set of models at every instant and apply the output of the corresponding controller to the plant. Since the number of available models is finite but the number of possible models is generally infinite, the identification is performed in two steps:

- The model with smallest error with respect to a criterion is rapidly chosen (switching).

- The parameters of the model are adjusted using a parameter adaptation algorithm (tuning).

The block diagram of this method is presented in Figure 3. The input and output of the plant are $u(t)$ and $y(t)$, respectively. The control system contains $n$ models $G_{1}, \ldots, G_{n}$ which are either fixed or adaptive models. The identification error is defined as the difference between the output $\hat{y}_{i}$ of the model $G_{i}$ and the plant output:

$$
\varepsilon_{i}=y(t)-\hat{y}_{i}(t)
$$




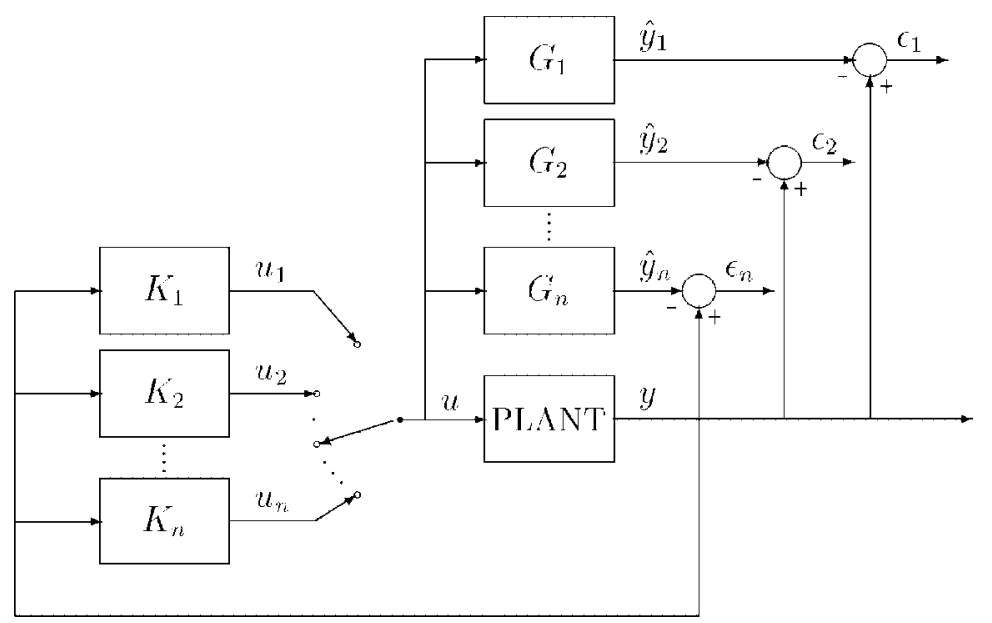

Figure 3. Schematic diagram of the multiple models approach.

For each model $G_{i}$, there is a controller $K_{i}$ that satisfies the control objective for $G_{i}$ (instead of $n$ controller we may have a parameterized controller $\left.K\left(G_{i}\right)\right)$. The performance criterion $J_{i}(t)$ which is used as the switching rule may be defined as follows [8]:

$$
J_{i}(t)=\sum_{j=0}^{t} \mathrm{e}^{-\lambda(t-j)} \varepsilon_{i}^{2}(j), \quad \lambda>0
$$

where $j$ is the time index and $\lambda$ is a forgetting factor which also assures the boundedness of the criterion for bounded $\varepsilon_{i}(t)$. Then one of the design parameters for the switching part of the control system is $\lambda$. Another design parameter $T_{\mathrm{d}}$ (dwell time), the minimum time delay between two switchings, plays an important role on the stability analysis of the system. In the sequel, we study the effect of these parameters on the performance of the system.

The models in this approach may be either fixed or adaptive. The parameters of the adaptive models may be initialized with the parameters of the last chosen fixed model in order to improve the adaptation speed. The stability analysis for several combinations of the models (all adaptive models, all fixed models, fixed models and one adaptive model, fixed models with one freerunning and one reinitialized adaptive model) was given in Reference [8] for the continuous time system and in Reference [11] for the discrete-time systems.

\section{CLOSED-LOOP OUTPUT ERROR ADAPTATION ALGORITHM}

The CLOE recursive adaptation algorithm (CLOE) presented in Reference [12] is based on a reparameterized adjustable predictor for the closed-loop system in terms of a known fixed controller $K$ and an adjustable plant model $\hat{G}$. Figure 4 shows the block diagram which is often used in closed-loop identification. 


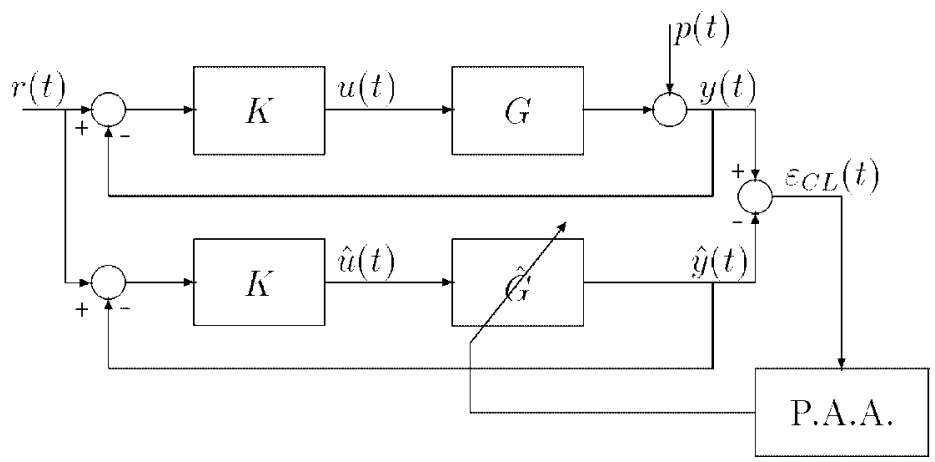

Figure 4. Closed-loop output error identification scheme.

The main advantages of this algorithm with respect to the classical recursive least-squares (RLS) algorithm are as follows:

- The parameter estimates are unbiased in the presence of noise when the identified model is in the model set [12].

- The frequency distribution of the modeling error is weighted by two sensitivity functions when the identified model is not in the model set. This leads to identify a suitable model for robust control design [13].

- The use of this algorithm in indirect adaptive control removes the need for adaptation freezing [9].

This algorithm can be summarized as follows [12]. Consider the output of the system which can be described by

$$
y(t)=\frac{q^{-\mathrm{d}} B\left(q^{-1}\right)}{A\left(q^{-1}\right)} u(t)+p(t)
$$

where $p(t)$ is the noise, assumed to be zero-mean with finite variance and independent with respect to the external excitation. Therefore,

$$
y(t+1)=\theta^{\mathrm{T}} \varphi(t)+A p(t+1)
$$

where

$$
\begin{aligned}
\theta^{\mathrm{T}} & =\left[a_{1} \cdots a_{n_{A}}, b_{1} \cdots b_{n_{B}}\right] \\
\varphi^{\mathrm{T}}(t) & =\left[-y(t), \cdots,-y\left(t-n_{A}+1\right), u(t-d), \cdots, u\left(t-n_{B}+1-d\right)\right] \\
u(t) & =K\left(q^{-1}\right)[r(t)-y(t)]
\end{aligned}
$$


The a priori and a posteriori predictors for the plant output are respectively, defined by:

$$
\begin{aligned}
\hat{y}^{\circ}(t+1) & =\hat{\theta}^{\mathrm{T}}(t) \phi(t) \\
\hat{y}(t+1) & =\hat{\theta}^{\mathrm{T}}(t+1) \phi(t)
\end{aligned}
$$

where

$$
\begin{aligned}
\hat{\theta}^{\mathrm{T}}(t) & =\left[\hat{a}_{1}(t) \cdots \hat{a}_{n_{A}}(t), \hat{b}_{1}(t) \ldots \hat{b}_{n_{B}}(t)\right] \\
\phi^{\mathrm{T}}(t) & =\left[-\hat{y}(t) \cdots-\hat{y}\left(t-n_{A}+1\right), \hat{u}(t-d) \ldots \hat{u}\left(t-n_{B}+1-d\right)\right] \\
\hat{u}(t) & =K\left(q^{-1}\right)[r(t)-\hat{y}(t)]
\end{aligned}
$$

Then the parameter adaptation algorithm has the form

$$
\begin{gathered}
\hat{\theta}(t+1)=\hat{\theta}(t)+F(t) \phi(t) \varepsilon_{\mathrm{CL}}(t+1) \\
F^{-1}(t+1)=\lambda_{1}(t) F^{-1}(t)+\lambda_{2}(t) \phi(t) \phi^{\mathrm{T}}(t) \\
0<\lambda_{1}(t) \leqslant 1, \quad 0 \leqslant \lambda_{2}(t)<2 \\
\varepsilon_{\mathrm{CL}}(t+1)=\frac{y(t+1)-\hat{y}^{\circ}(t+1)}{1+\phi^{\mathrm{T}}(t) F(t) \phi(t)}
\end{gathered}
$$

The stability and the convergence analysis of this algorithm have been given in Reference [12]. According to the analysis, the positive realness of a closed-loop transfer function $(S / P$, where $S$ is the denominator of the controller and $P$ is the characteristic polynomial of the closed loop) plays an important role on the stability and the convergence of the algorithm. However, this positive real condition may be weakened using a filtered regression vector defined as

$$
\phi_{\mathrm{f}}(t)=\frac{S}{\widehat{P}} \phi(t)
$$

where $\hat{P}=\hat{A} S+q^{-\mathrm{d}} \hat{B} R$ is an estimation of the closed-loop polynomial based on an estimation of $A$ and $B$. One uses the parameter adaptation algorithm of Equations (16)-(19) in which $\phi(t)$ is replaced by $\phi_{\mathrm{f}}(t)$. This algorithm named F-CLOE [12] will be used in the simulation examples of Section 5 because in this case the transfer function $\hat{P} / P$ should be positive real which is much milder than the positive real condition on $S / P$. In these simulations $\hat{P}$ is in fact the desired closed-loop polynomial used for the pole placement method.

The schematic diagram of the indirect adaptive control using CLOE method is compared with the classical indirect adaptive control using the RLS parameter adaptation algorithm in Figure 5.

A preliminary study [14] shows that the existence of an excitation signal is necessary for the stability of an adaptive control system using the CLOE algorithm in a certain situation (when the closed-loop system becomes unstable in the absence of external excitation). However, this algorithm can be used in the multiple models scheme even if the stability condition for the algorithm is not satisfied. The reason is that in the absence of the excitation signal the parameters 


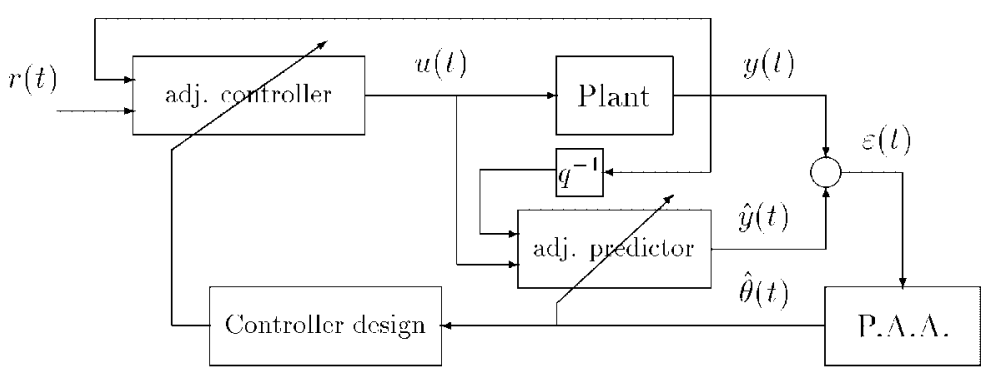

(a)

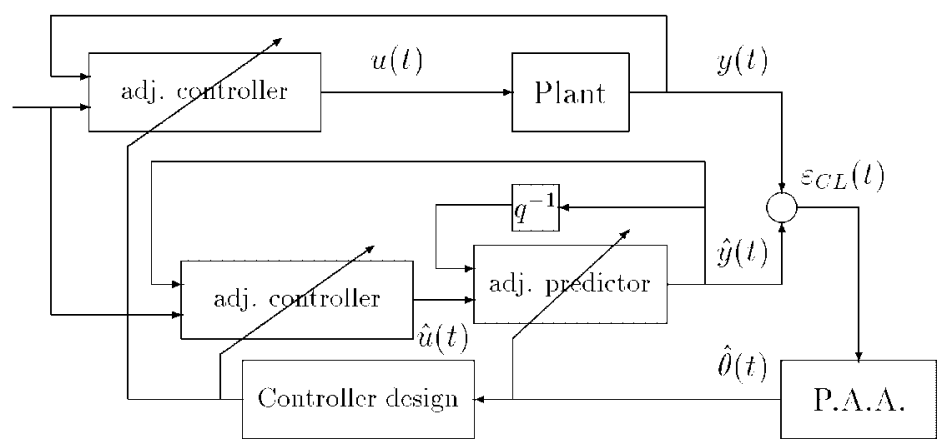

(b)

Figure 5. Indirect adaptive control (a) classical adaptive control (RLS); (b) CLOE adaptive control.

of the CLOE predictor remain unchanged and the adaptive model becomes a fixed model among the other fixed models, for which a stability analysis has been already presented $[8,11]$.

\section{SIMULATION RESULTS}

In this section, several simulation examples will be performed in order to show the effects of the design parameters on the performance of the flexible transmission system. The simulations are carried out by VisSim [15] software. For this purpose, different functions have been developed in order to realize the multimodel adaptive control on this software.

Selection of appropriate values for the design parameters depends upon some information about the plant, like:

- plant model in different operating points,

- speed of parameter variations of the plant model,

- existence and type of the reference signal,

- existence and type of the output disturbances,

- variance of the output noise.

For the flexible transmission system, in order to simulate the above-mentioned characteristics, 13 discrete-time identified models of plant relating to different loadings are considered. Then we 
suppose that plant is initially unloaded and the small disks are placed on the third pulley one by one until the system becomes full loaded (with 12 disks). Next, the disks are taken off one by one and system again becomes unloaded. It is supposed that this load changing is repeated cyclically with period $T_{\mathrm{c}}$ and we refer to $f_{\mathrm{c}}=1 / T_{\mathrm{c}}$ as the parameter changing rate. Therefore, a small value for $f_{\mathrm{c}}$ indicates a system with non-frequent (spaced) parameter variations and a large value for $f_{\mathrm{c}}$ simulates a system with frequent and large parameter variations. The reference signal is either null or a filtered square wave signal (filtered by a reference model) with an amplitude of 1 and a period of $10 \mathrm{~s}$. The output disturbance signal is also either null or a pulse train with an amplitude of 0.5 and a period of $20 \mathrm{~s}$. A zero-mean normally distributed white noise is added to the plant output. The noise variance is varied in different simulations to study the noise effect.

The objective of the control system is to follow the reference input and to reject the output disturbances as fast as possible. Thus, in order to compare different design parameters a performance index is defined as follows:

$$
J_{\mathrm{c}}=\left(\frac{1}{T_{\mathrm{f}}} \int_{0}^{T_{\mathrm{f}}} \varepsilon_{\mathrm{c}}^{2}(t) \mathrm{d} t\right)^{1 / 2}
$$

where

$$
\varepsilon_{\mathrm{c}}(t)=r(t)-y(t)
$$

and $T_{\mathrm{f}}$ is the simulation time. The performance index is in fact the root-mean-square of tracking error and since all the controllers contain an integrator and disturbances are applied while $r(t)=0$ it represents the root-mean-square of regulation error as well.

Design of a multimodel adaptive control system consists of the following steps:

(1) Determine the number of fixed and adaptive models.

(2) Choose the adaptation algorithm (RLS or CLOE).

(3) Determine the forgetting factor $\lambda$.

(4) Determine the minimum time between switchings $T_{\mathrm{d}}$.

(5) Choose a controller for each model or determine a control law based on the model parameters.

In this paper, we are not going to discuss about the design of the controllers (step (5)), although it affects significantly the overall performance of the system. In fact, we suppose that readers are able to design a controller for a fixed model which satisfies the specifications. However, in the simulations we use a two-degree-of-freedom digital robust controller designed by the pole placement with sensitivity function shaping method described in Reference [9]. This controller is robust with respect to additive uncertainties and contains an integrator to reject constant disturbances and to obtain a zero steady-state error.

In what follows, we show how the performance of a multimodel adaptive control system is related to the design parameters.

\subsection{Number of fixed and adaptive models}

The first step in the multimodel control design is to determine the number of fixed and adaptive models. The number of fixed models may be chosen equal to the number of operating points. 
Principally, better performances will be achieved with more fixed models. However, the price is a more complex control system which leads to more computation time and less reliability. It should be mentioned that if a robust control design is considered, one robust controller may give a good performance for different operating points and can reduce the number of fixed models. An adaptive model can also reduce the number of fixed models under the following conditions:

(1) There exists an excitation signal on the reference input.

(2) The abrupt changes of parameters are sufficiently spaced in the time (i.e. there is enough time between two changes for parameter adaptation).

Therefore for the systems in regulation (with a fixed reference signal) adaptive models should not be used in the models set.

We may use several adaptive models when the plant models in different operating points have different structures (orders). In such cases, for each structure one adaptive model with appropriate number of parameters may be employed.

The following simulations show that when the parameter variations are sufficiently spaced, one adaptive model can reduce the number of fixed models without changing in the overall performance. But when the parameter variations are frequent, an adaptive model has less effect.

5.1.1. Spaced parameter variations. In this simulation example, it is supposed that the period of the cyclic changes in the model parameters is $T_{\mathrm{c}} \geqslant 480 \mathrm{~s}$ (there is at least $20 \mathrm{~s}$ between the parameter variations). For the models set of the control system three combinations are considered as follows:

(1) Two fixed models (no. 0,9).

(2) Seven fixed models (no. 0,2,4,6,8,10,12).

(3) Two fixed and one adaptive models (no. 0,9).

The schematic diagram of the third case is shown in Figure 6. $G_{1}$ and $G_{2}$ represent the fixed models no. 0 and 9 , respectively, and $\hat{G}$ is the adaptive model with reinitialization using the CLOE algorithm. At every instant, the supervisor chooses the best model $G^{*}$ according to the performance index of Equation (5) and the control input $u(t)$ is determined based on this model and using the pole placement method. An estimation of the control input $\hat{u}(t)$ is also computed which will be used to determine $\hat{y}(t)$ and $\varepsilon_{C L}(t)$ in the CLOE algorithm. It should be mentioned that the adaptive free running model which is theoretically needed for deriving a stability proof was not necessary in this simulation because there exists always a fixed model which leads to a stabilizing controller for all of the loadings.

The parameter of the switching rule are: $\lambda=0.05$ and $T_{\mathrm{d}}=1$ sampling period $(50 \mathrm{~ms})$. The simulation time $T_{\mathrm{f}}$ is $1440 \mathrm{~s}$. The performance index $J_{\mathrm{c}}$ versus the parameter changing rate $\left(f_{\mathrm{c}}=1 / T_{\mathrm{c}}\right)$ is plotted for the three cases in Figure 7. One can observe that the performance is improved when the number of fixed models is increased. It should be mentioned that the performance cannot be improved significantly using more than seven fixed models, because the controllers are robust and give also a good performance even when the plant model is not among the fixed models of the control system. It is also observed that in the third case (one adaptive model and two fixed models) we have almost the same performance as the second case (seven fixed models) which shows that one adaptive model can replace five fixed models. 


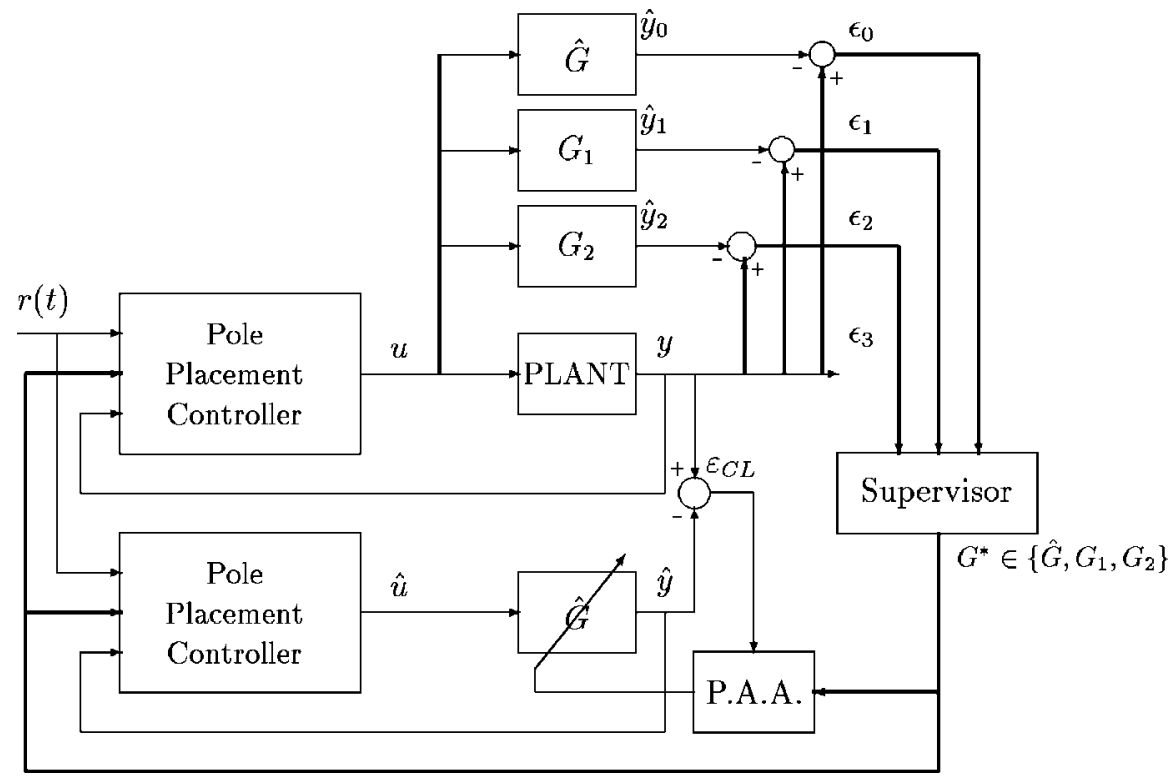

Figure 6. Bloc diagram of the multimodel adaptive control (case 3).

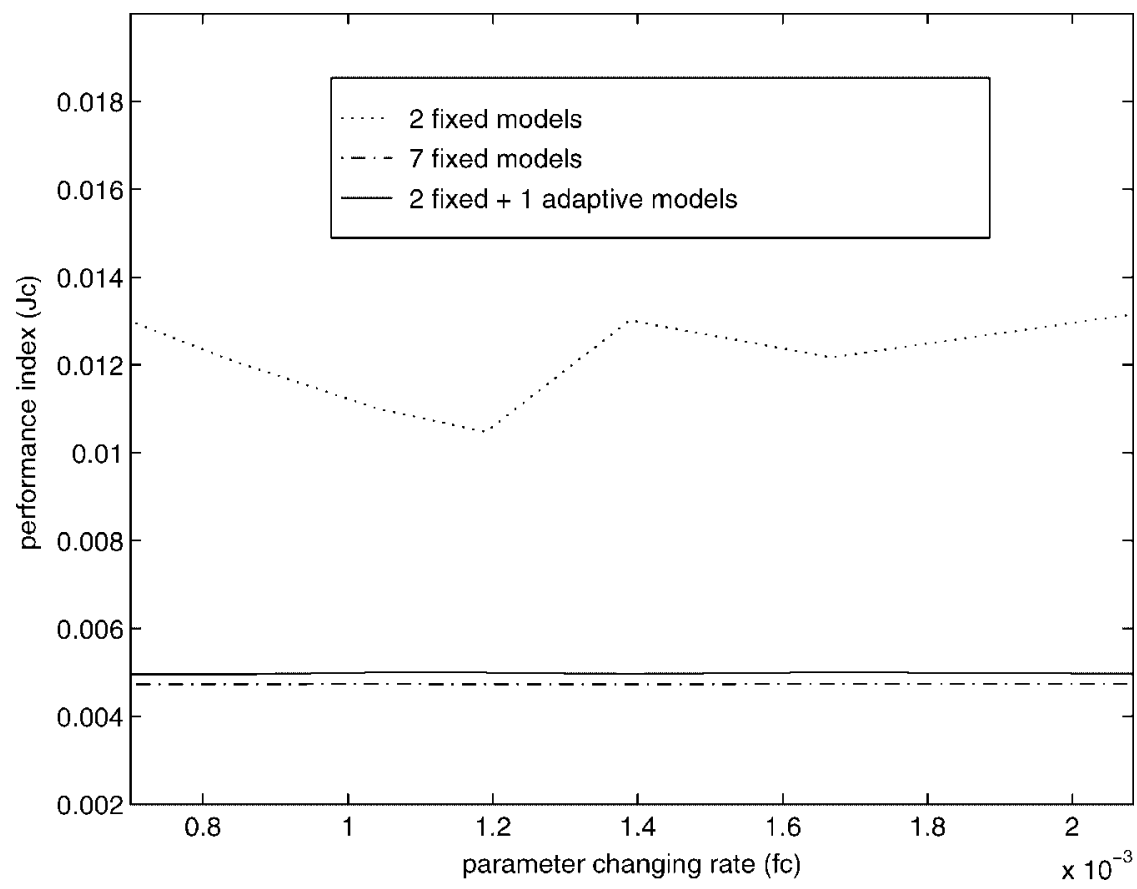

Figure 7. Performance index versus parameter changing rate (spaced parameter variations). 
5.1.2. Frequent parameter variations. This simulation example is performed under the same condition as the last simulation with the difference that the period $T_{\mathrm{c}}$ of the parameter cyclic changes is between 20 and $100 \mathrm{~s}\left(20 \leqslant T_{\mathrm{c}} \leqslant 100\right)$ which indicates very frequent parameter variations. A shorter simulation time $T_{\mathrm{f}}=120 \mathrm{~s}$ and a greater forgetting factor $\lambda=0.1$ are also chosen. Figure 8 depicts that in this case an adaptive model does not change so much the performances of the system. The reason is that, the frequent parameter variations of the plant cause rapid switching between the models of the control system and the adaptive model has not enough time to adapt their parameters between two switchings. Thus, in such a situation only fixed models should be employed. The non-monotonic character of the curve is caused by the very complex relation between the frequency of parameter change and the performance index. In fact, it is possible that the performance be randomly improved when $f_{\mathrm{c}}$ is increased. Consider the case that a number of parameter changes occur when the reference signal is constant, these changes will not be detected by the control system and the system output remains constant which leads to improve the performance index. However, it is the average character of the performance index which may be used for the conclusions.

\subsection{Parameter adaptation algorithm}

The second step of the multimodel adaptive control design is to choose the type of adaptation algorithm for the adaptive model (or models). In this section, we will show that the CLOE adaptation algorithm gives better performances than the classical RLS algorithm in the presence of noise. We consider three fixed $(0,6,12)$ and one adaptive model in the models set of the control

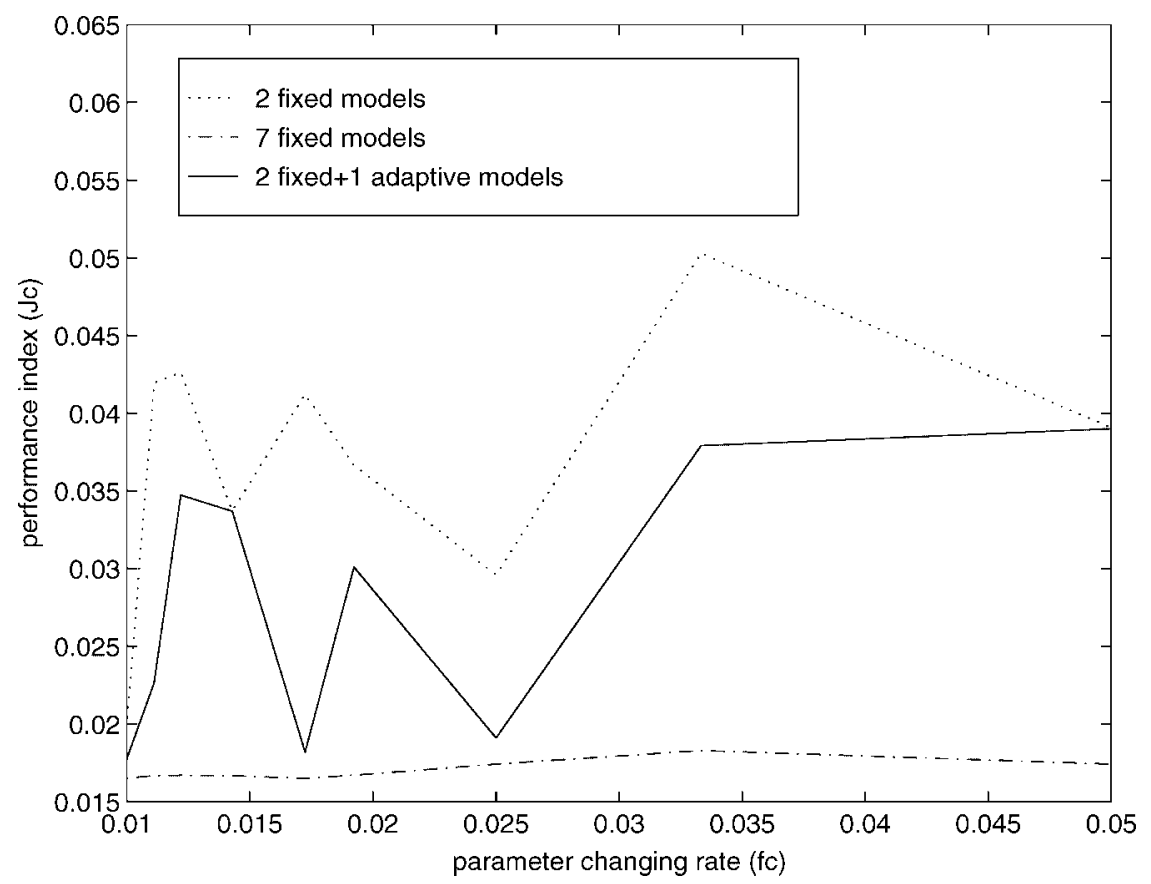

Figure 8. Performance index versus parameter changing rate (frequent parameter variations). 
system. Two distinct simulations are carried out, one using CLOE algorithm in the adaptive model and the other with RLS adaptation algorithm. The plant model is supposed to be fixed on the model no. 3 which does not belong to the fixed models of the control system. Therefore, the switching will be stopped after a time on the adaptive model and the parameters of the adaptive model will be tuned by the adaptation algorithm. The parameters of the switching part are chosen as follows: $\lambda=0.05$ and $T_{\mathrm{d}}=1 \mathrm{sampling}$ period. The simulation time $T_{\mathrm{f}}$ is $120 \mathrm{~s}$. The variance of the output noise is increased from 0 up to 0.07 and the performance index of the system is plotted versus the noise variance in Figure 9. As depicted in this figure, increasing the noise variance will deteriorate the system performances in both cases, but CLOE algorithm gives better performances especially when the noise variance is high.

Figure 10 shows the output, the reference and the switching diagram for this simulation using the CLOE algorithm with a noise variance of 0.07 . The switching diagram shows the best model chosen by the supervisor at each instant. In this diagram 0 corresponds to the adaptive model and 1,2 and 3 correspond, respectively, to model no. 0,6 and 12. This figure can be compared with Figure 11 corresponding to the RLS algorithm. It can be observed that the larger variations of the output (using RLS algorithm) lead to the unwanted switchings which consequently deteriorate the performances.

\subsection{Forgetting factor $\lambda$}

The forgetting factor $\lambda$ in the switching rule plays an important role on the performance of the control system. The speed of parameter changes, the variance of output noise and the type of

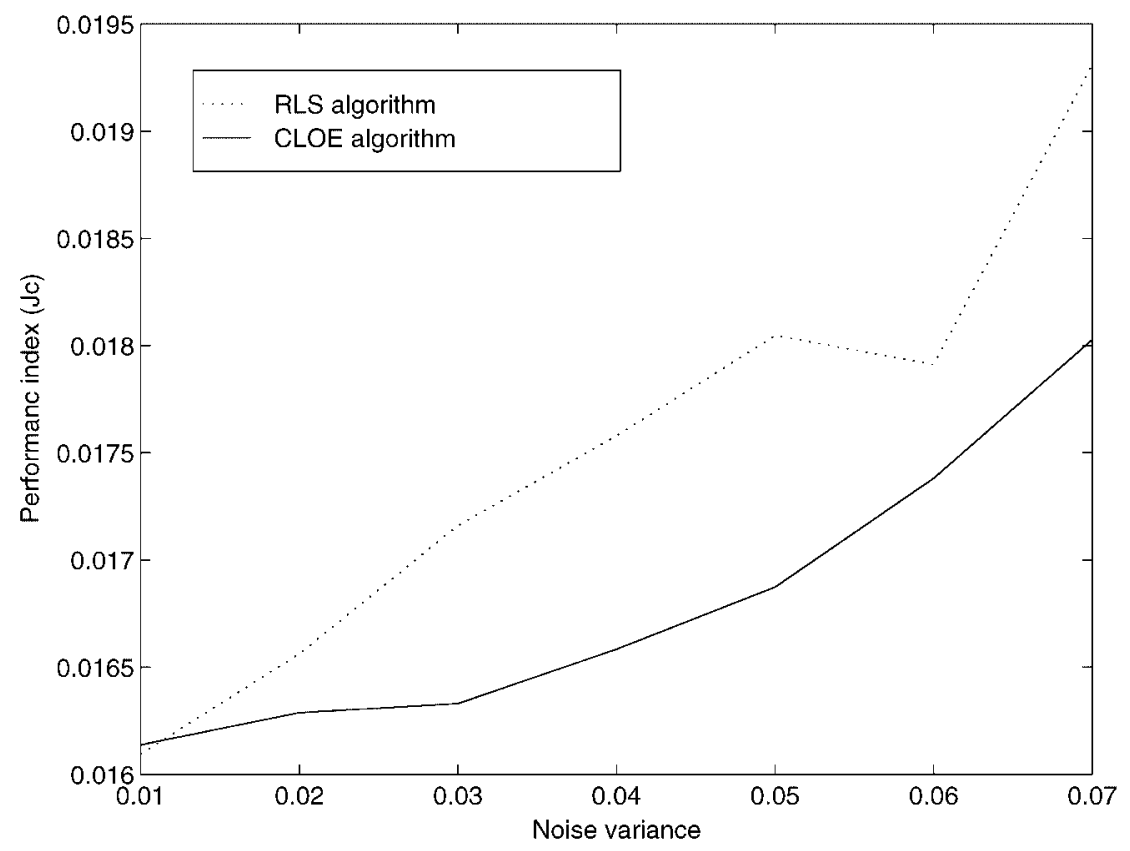

Figure 9. Performance index versus noise variance (comparison of the adaptation algorithms). 

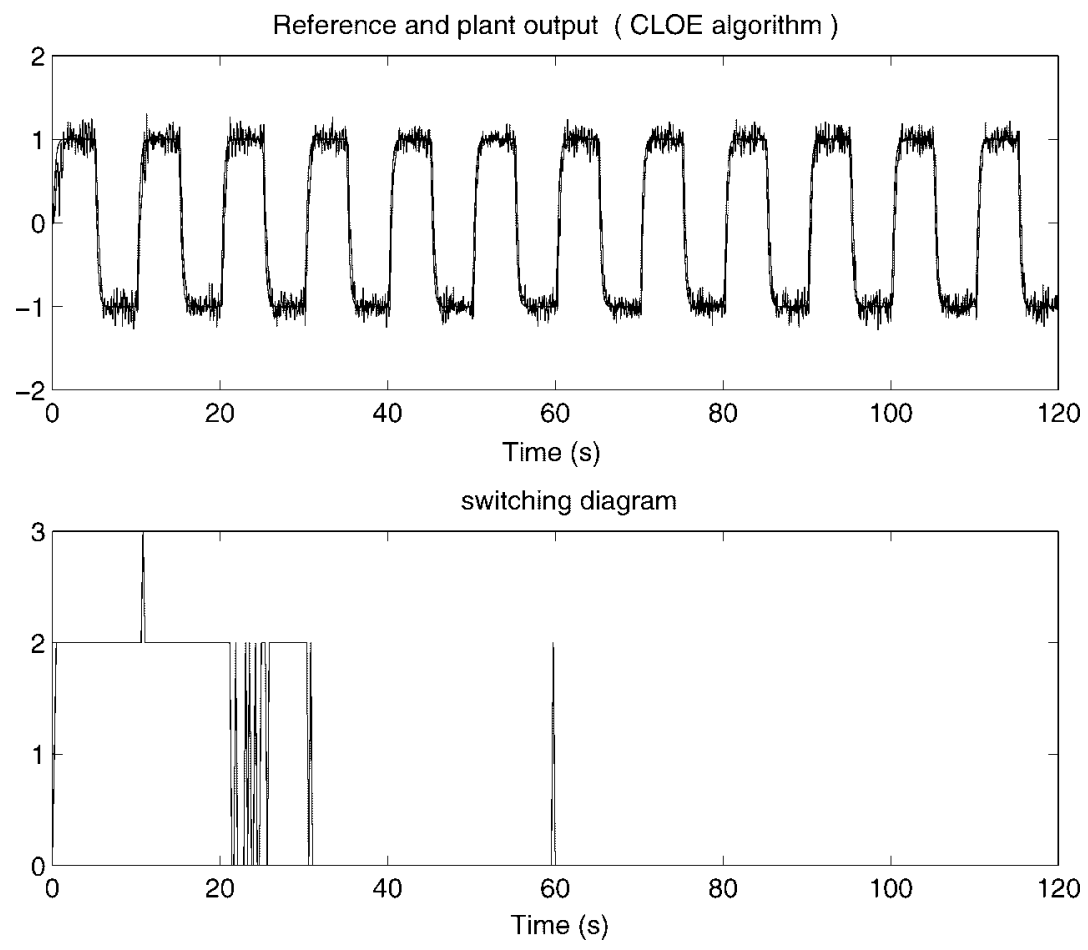

Figure 10. Simulation results using the CLOE adaptation algorithm.

output disturbances affect the choice of $\lambda$. In order to study these effects four simulations are performed. Like the preceding simulation example three fixed models (no. 0,6,12) and one adaptive model (using CLOE adaptation algorithm) are considered in the set of models of the control system. The dwell time is $T_{\mathrm{d}}=1$.

5.3.1. Spaced parameter variations. In this part, we choose the period of the parameter changes $T_{\mathrm{c}}=480 \mathrm{~s}$ which represents spaced parameter variations. The simulation results for $T_{\mathrm{f}}=480 \mathrm{~s}$ are presented in Figure 12(a). One can observe that for this type of parameter variations we should select a small value for $\lambda$. Because for small $\lambda$ the switching criterion approaches to a model identification criterion which leads to select the best model among the models set for the plant.

5.3.2. Frequent parameter variations. In order to simulate frequent and fast parameter variations, the period of the parameter changes is chosen equal to $20 \mathrm{~s}$, that means the model of the plant changes from model no. 0 to 12 and return to 0 in 20 s. In Figure 12(b) the performance index $J_{\mathrm{c}}$ for $T_{\mathrm{f}}=120 \mathrm{~s}$ is plotted for different values of $\lambda$. It clearly shows that the larger values for $\lambda$ lead to the better performances of the system. The reason is that for a large value of $\lambda$ the latest errors have more weightings in the switching criterion which cause a very quick response to the abrupt parameter changing. 

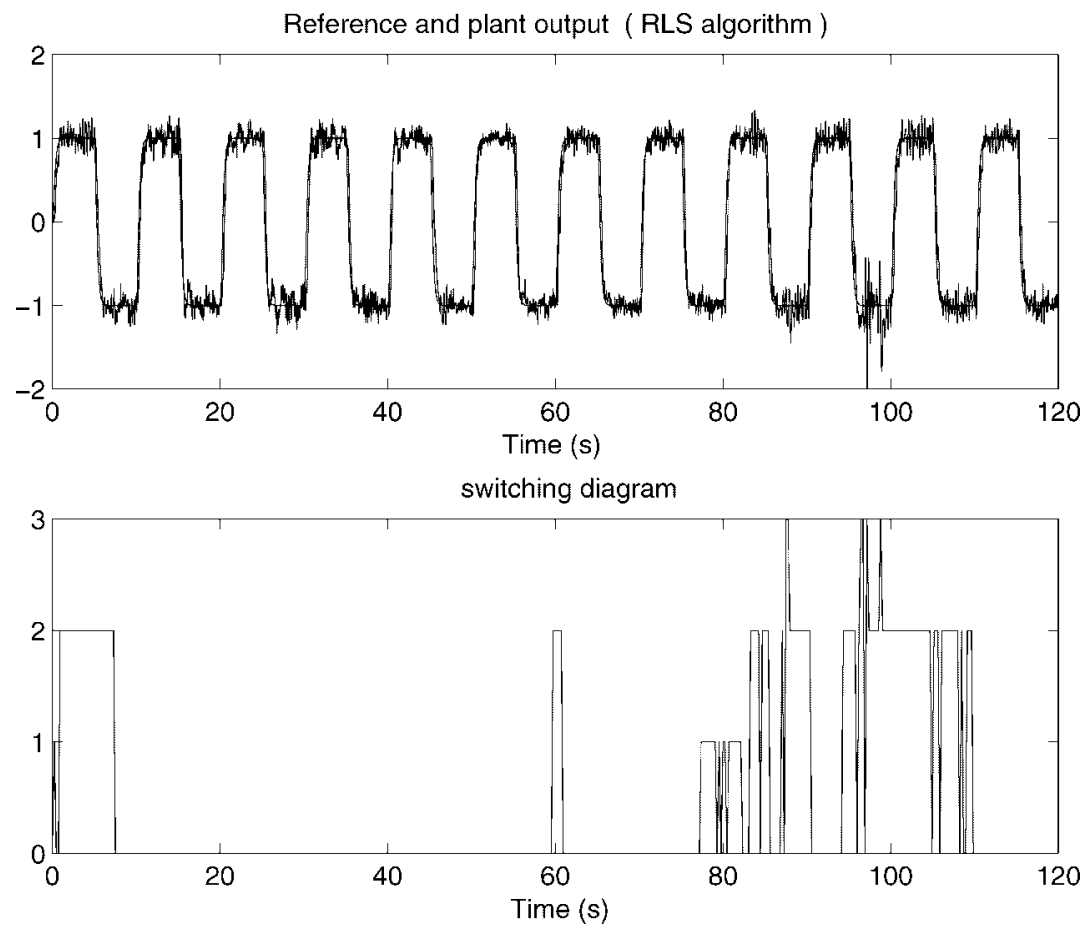

Figure 11. Simulation results using the RLS adaptation algorithm.

5.3.3. Disturbance effect. The output disturbance is modelled as a square wave signal with 0.5 amplitude and $20 \mathrm{~s}$ period added to the output of the plant. The plant model is fixed (model no. 3) and the parameters of the switching part is the same as preceding simulations. The simulation results for $T_{\mathrm{f}}=100 \mathrm{~s}$ (Figure 12(c)) illustrate that smaller $\lambda$ prevents the unwanted switchings and rejects better the disturbances.

5.3.4. Noise effect. In order to study the noise effect on the performance of the control system, the plant model is fixed to the model no. 3 and the noise variance is 0.1. It is shown in Figure 12(d) that for a noisy system $\lambda$ should be kept small in order to avoid the unwanted switchings. A large value for $\lambda$ makes the switching criterion very sensitive to noise and leads to poor performances.

It should be noticed that the choice of $\lambda$ for a noisy system with spaced parameter variations subject to output disturbance is non-conflicting, but for a system with frequent and large parameter variations is conflicting. In such situations the choice of $\lambda$ should be performed with precautions. However, experiences shows that a large value (greater than 0.5 ) for $\lambda$ should be avoided.

\subsection{Dwell time $T_{\mathrm{d}}$}

The effect of the minimum time between two consecutive switchings $T_{\mathrm{d}}$ on the performance of the system is studied via two simulations. In the first one we have frequent parameter variations and 
(a) spaced variations

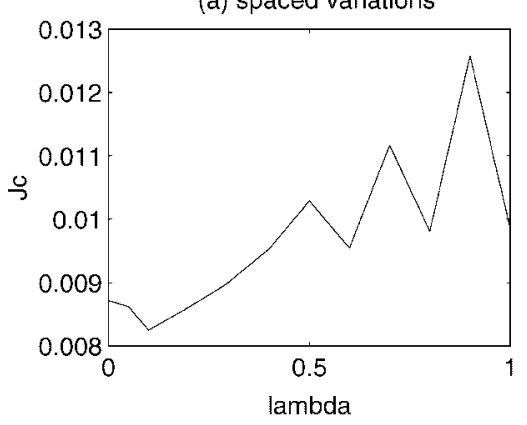

(c) output disturbance

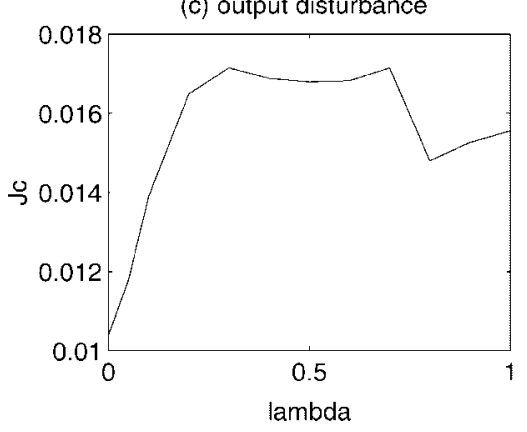

(b) frequent variations

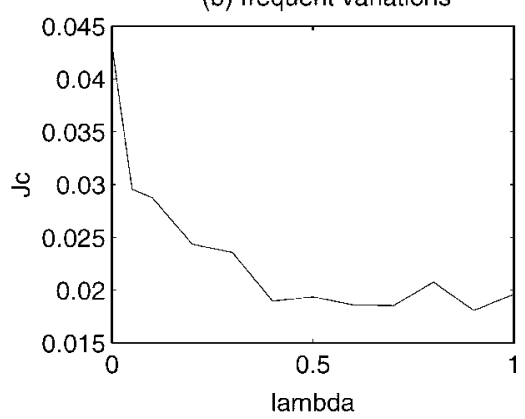

(d) noisy environment

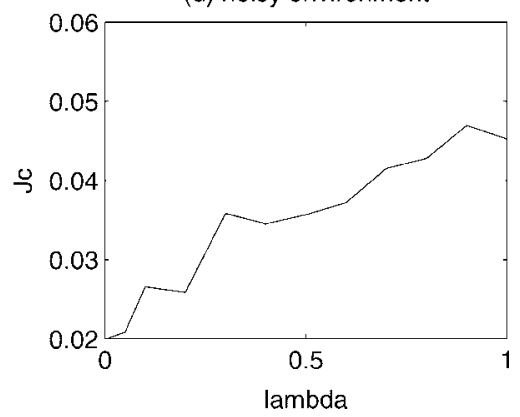

Figure 12. Performance index versus $\lambda$.

in the second the parameter variations are spaced. For the frequent parameter variations we choose $T_{\mathrm{c}}=20 \mathrm{~s}$ and $T_{\mathrm{f}}=120 \mathrm{~s}$ and for the spaced parameter variations $T_{\mathrm{c}}=480 \mathrm{~s}$ and $T_{\mathrm{f}}=480 \mathrm{~s}$. The performance index $J_{\mathrm{c}}$ is plotted versus $T_{\mathrm{d}}$ increasing from 1 up to 30 sampling period. The forgetting factor $\lambda$ is fixed to 0.1 for both cases. The simulation results of Figure 13 shows that a large value for $T_{\mathrm{d}}$ deteriorates the performances when the parameter variations are frequent and has almost no effect when the parameter variations are spaced.

\section{CONCLUSIONS}

The design parameters selection of multimodel adaptive control has been investigated via several simulations for a flexible transmission system. The effects on the overall system performance of number of fixed and adaptive models, type of adaptation algorithm, forgetting factor and dwell time in the switching part have been studied. The results of this study can be summarized as follows:

- An adaptive model can reduce the number of fixed models of the control system if there exists an excitation signal and enough time for parameter adaptation in the tuning phase. For the system operating in regulation and/or with short tuning phase adaptive models are less effective. 


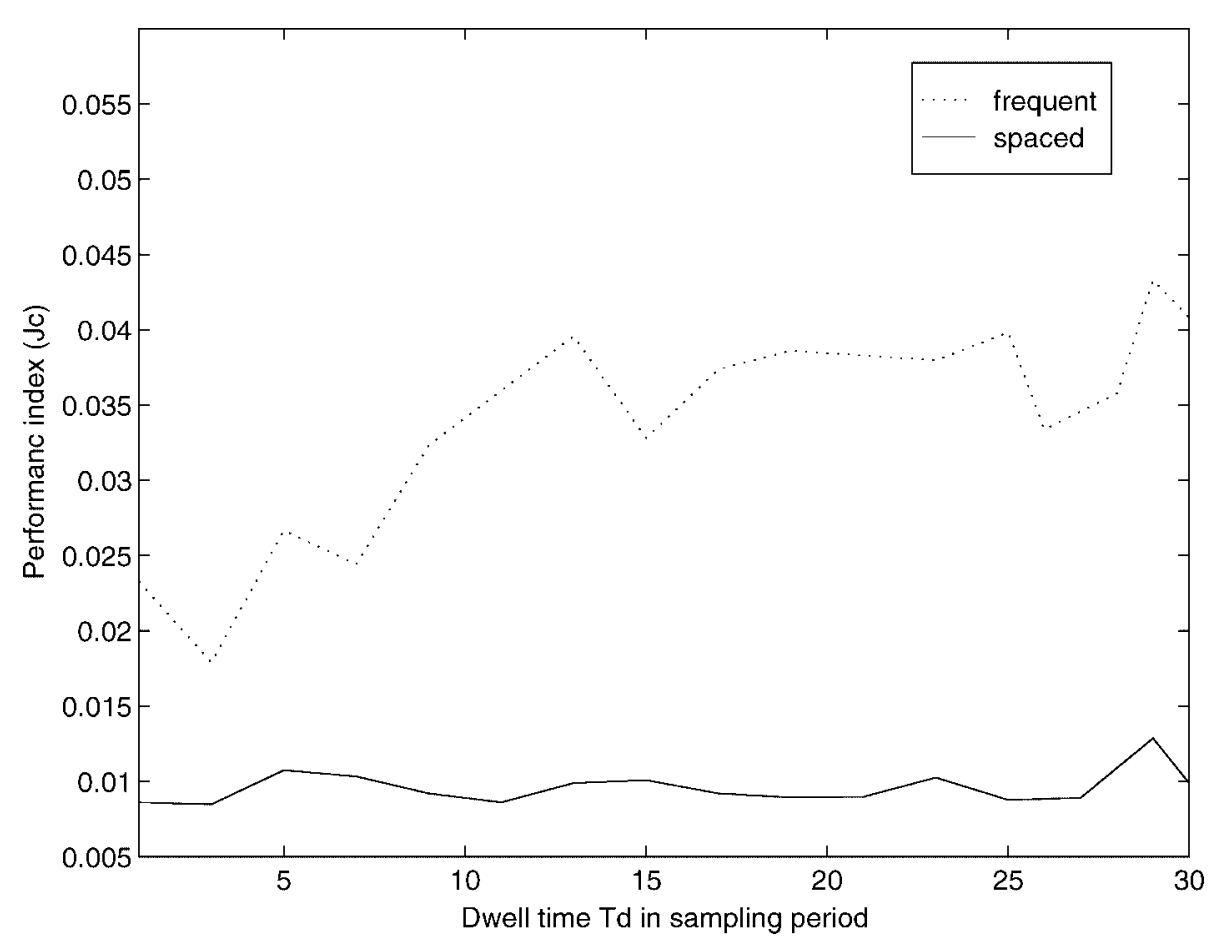

Figure 13. Performance index versus dwell time $T_{\mathrm{d}}$.

- A new parameter adaptation algorithm based on CLOE gives better performances in the tuning phase than the classical RLS adaptation algorithm in the presence of noise.

- Selection of the forgetting factor in the switching criterion is probably the most crucial step of the control design. The speed of parameter changes, the noise variance and the output disturbance influence the choice of the forgetting factor. A compromise between noise and disturbance rejection and fast parameter adaptation should be performed for the systems with frequent parameter variations subject to noise and output disturbances.

- The dwell time should be kept as small as possible when the parameter variations are frequent. For the spaced parameter variations the dwell time has almost no effect on the performance. It should be mentioned that in some situations (while the output disturbances and noise are significant) $T_{\mathrm{d}}$ may be increased in order to reduce the unwanted switchings and thus improve the stability.

\section{REFERENCES}

1. Martensson B. Adaptive stabilization. Ph.D. Thesis, Lund Institute of Technology, Lund, Sweden, 1986.

2. Fu M, Barmish BR. Adaptive stabilization of linear systems via switching control. IEEE Transactions on Automatic Control 1986; 31:1097-1103.

3. Miller DE, Davison EJ. An adaptive controller which provides Lyapunov stability, IEEE Transactions on Automatic Control, 1989; 34:599-609.

4. Miller DE. Adaptive stabilization using a nonlinear time-varying controller. IEEE Transactions on Automatic Control 1994; 39:1347-1359.

5. Morse AS. Control using logic-based switching. In Trends in Control, Isidori A. (Ed.). Springer: Heidelberg, 1995. 
6. Morse AS, Mayne DQ, Goodwin GC. Application of hysteresis switching in parameter adaptive control. IEEE Transactions on Automatic Control 1992; 37:1343-1354.

7. Narendra KS, Balakrishnan J. Improving transient response of adaptive control systems using multiple models and switching. IEEE Transactions on Automatic Control 1994; 39:1861-1866.

8. Narendra K, Balakrishnan. Adaptive control using multiple models IEEE Transactions on Automatic Control 1997; 42(2):171-187.

9. Karimi A, Landau ID. Robust adaptive control of a flexible transmission system using multiple models. Proceedings of the 37th CDC, Tampa, Florida USA, December 1998.

10. Landau ID, Rey D, Karimi A, Voda A, Franco A. A flexible transmission system as a benchmark for robust digital control. European Journal of Control 1995; 1(2).

11. Narendra KS, Xiang G. Adaptive control of discrete-time systems using multiple models. Proceedings of the 37th $C D C$, Tampa, Florida USA 1998.

12. Landau ID, Karimi A. An output error recursive algorithm for unbiased identification in closed loop. Automatica 1997; 33(5):933-938.

13. Karimi A, Landau ID. Comparison of the closed loop identification methods in terms of the bias distribution. Systems and Control Letters 1998; 34:159-167.

14. Karimi A. Conception des régulateurs numériques robustes et adaptatifs Ph.D. Thesis, Institut National Polytechnique de Grenoble, LAG, Grenoble, France, 1997.

15. Visual Solutions, Inc., 487 Groton Rd. Westford, MA USA, VisSim Users's Guide, 1996. 